\title{
Poincaré invariance and the heavy-quark potential
}

\author{
Nora Brambilla* \\ Dipartimento di Fisica, Università degli Studi di Milano, via Celoria 16, 20133 Milano, Italy \\ Dieter Gromes ${ }^{\dagger}$ \\ Institut für Theoretische Physik, Universität Heidelberg, Philosophenweg 16, 69120 Heidelberg, Germany \\ Antonio Vairo \\ Theory Division, CERN, 1211 Geneva 23, Switzerland \\ (Received 9 April 2001; published 11 September 2001)
}

\begin{abstract}
We derive and discuss the constraints induced by Poincare invariance on the form of the heavy-quark potential up to order $1 / \mathrm{m}^{2}$. We present two derivations: one uses general arguments directly based on the Poincare algebra and the other follows from an explicit calculation on the expression of the potential in terms of Wilson loops. We confirm relations from the literature, but also clarify the origin of a long-standing false statement pointed out recently.
\end{abstract}

DOI: 10.1103/PhysRevD.64.076010

PACS number(s): 11.30.Cp, 12.38.Lg, 12.39.Hg

\section{INTRODUCTION}

The effective field theory (EFT) approach has recently clarified under which circumstances the heavy-quarkantiquark interaction may be described by a simple potential picture. We call potential nonrelativistic QCD (pNRQCD) the corresponding EFT. Moreover, in this framework the complete $1 / \mathrm{m}^{2}$ quark-antiquark potential has been derived $[1,2]$. The final expression of the potential is given in terms of Wilson loops [3] with field insertions. Therefore, it is appropriate also in the situation where the quark-antiquark interaction is non-perturbative. In this case the Wilson loop operators may be calculated on the lattice [4] or in QCD vacuum models [5].

With respect to the classical literature on the subject (for some reviews see [6,7]) the derivation $[1,2]$ presents the following improvements. (i) Being done in an EFT framework it has been proved, at least at the present accuracy of the theory, to correctly implement the ultraviolet behavior of QCD. This is encoded into the matching coefficients inherited from the matching to NRQCD [8] (on the relation between the matching coefficients of NRQCD and the heavyquark potential see also [9]). (ii) In addition to the spin and momentum-dependent potentials, it includes the $1 / \mathrm{m}$ and $1 / \mathrm{m}^{2}$ momentum and spin-independent potentials that have not been calculated before $[10,11]$. Hence, the obtained expression is complete under the conditions specified in $[1,2]$. (iii) It corrects some errors present in the previous literature, which have been propagated in several related papers until today (we refer to [2] for a discussion on this point).

In view of this new information (and in some case corrections) with respect to the previous knowledge on the subject,

\footnotetext{
*Email address: nora.brambilla@cern.ch

†Email address: d.gromes@ thphys.uni-heidelberg.de

‡Email address: antonio.vairo@cern.ch
}

one has to ask if other results, present in the literature and related to the form of the heavy-quark potential, may change. In particular in [12] and [13] exact relations among the spin and momentum-dependent potentials, respectively, have been derived. A more recent analysis can be found in [14]. One may wonder if the errors found in the previous literature and/or the new terms added to the potential spoil the validity of those exact relations. In the present paper we will address this question and derive ex novo, by general considerations on the structure of the potential and by explicit manipulations of the expressions given in [1,2], the relations induced by Poincare invariance on the form of the potentials. We shall confirm the results found in $[12,13]$ and explain why the errors found in the literature on the derivation of the potentials do not affect the derivation of the exact relations among them. Since the potentials are defined up to unitary transformations, we shall also show that the relations found are, indeed, invariant with respect to them.

The consequences of the above analysis, i.e., the obtained exact relations among the heavy-quark potentials, may be relevant in several situations. For instance, they allow us to have independent checks on analytic, and lattice computations. However, in our opinion, the most important application is to establish and constrain the power counting of NRQCD under the specific situations where this EFT may be substituted by pNRQCD. This is an important point not only in the study of the quarkonium spectrum, for instance by means of lattice NRQCD, but also in the study of the quarkonium production where the validity of the perturbative NRQCD power counting has been recently questioned $[2,15]$.

The paper is organized as follows. In Sec. II we briefly describe the physical situation we are considering and write down the corresponding pNRQCD Lagrangian. In Sec. III we discuss Poincaré invariance from a general point of view and derive relations among the different potentials without specifying their explicit form. In Sec. IV the previous relations are proven to be valid by direct computation on the potentials explicitly given in $[1,2]$. In Sec. V we discuss the 
obtained results. In an Appendix we clarify in some detail the origin of the error in the previous literature, which was found in [2], and its relation with the derivation of Sec. IV.

\section{II. $\mathrm{pNRQCD}$}

In this work we consider pNRQCD in the situation where the singlet field $S$, describing the heavy-quarkonium system, is the only available ultrasoft degree of freedom. ${ }^{1}$ In the perturbative regime this situation corresponds to considering pNRQCD at the leading order in the multipole expansion ${ }^{2}$ while in the non-perturbative one it corresponds to considering pNRQCD without light quarks and ultrasoft gluonic excitations under the circumstances discussed in $[1,2]$. In particular, it is assumed that all gluonic excitations have a gap of order $\Lambda_{\mathrm{QCD}}$ with respect to the singlet and are integrated out by matching NRQCD to pNRQCD. The pNRQCD Lagrangian reads

$$
\mathcal{L}_{\mathrm{pNRQCD}}=S^{\dagger}\left[i \partial_{0}-h_{s}\left(\mathbf{x}_{1}, \mathbf{x}_{2}, \mathbf{p}_{1}, \mathbf{p}_{2}\right)\right] S,
$$

where $\mathbf{x}_{j}$ and $\mathbf{p}_{j}$ are the position and the momentum operators of a heavy quark of mass $m_{j}$ and spin $\mathbf{S}_{j}$. The operator $h_{s}$ can be identified with the Hamiltonian of the singlet and has the following structure ${ }^{3}$ up to order $1 / \mathrm{m}^{2}$

$$
\begin{aligned}
h_{s}\left(\mathbf{x}_{1}, \mathbf{x}_{2}, \mathbf{p}_{1}, \mathbf{p}_{2}\right)= & \frac{\mathbf{p}_{1}^{2}}{2 m_{1}}+\frac{\mathbf{p}_{2}^{2}}{2 m_{2}}+V^{(0)}(r)+\frac{V^{(1,0)}(r)}{m_{1}} \\
& +\frac{V^{(0,1)}(r)}{m_{2}}+\frac{V^{(2,0)}}{m_{1}^{2}}+\frac{V^{(0,2)}}{m_{2}^{2}}+\frac{V^{(1,1)}}{m_{1} m_{2}}, \\
V^{(2,0)=} & \frac{1}{2}\left\{\mathbf{p}_{1}^{2}, V_{\mathbf{p}^{2}}^{(2,0)}(r)\right\}+\frac{V_{\mathbf{L}^{2}}^{(2,0)}(r)}{r^{2}} \mathbf{L}_{1}^{2}+V_{r}^{(2,0)}(r) \\
& +V_{L S}^{(2,0)}(r) \mathbf{L}_{1} \cdot \mathbf{S}_{1}, \\
V^{(0,2)=} & \frac{1}{2}\left\{\mathbf{p}_{2}^{2}, V_{\mathbf{p}^{2}}^{(0,2)}(r)\right\}+\frac{V_{\mathbf{L}^{2}}^{(0,2)}(r)}{r^{2}} \mathbf{L}_{2}^{2}+V_{r}^{(0,2)}(r) \\
& -V_{L S}^{(0,2)}(r) \mathbf{L}_{2} \cdot \mathbf{S}_{2},
\end{aligned}
$$

\footnotetext{
${ }^{1}$ In a non-relativistic bound state at least two energy scales are dynamically generated: the "soft" scale $m v$, which sets the scale of the inverse of the bound-state radius, and the "ultrasoft" scale $m v^{2}$ of the order of the binding energy. The velocity $v$ of the particle in the bound state is $v \ll 1$, so that the scales are hierarchically ordered. pNRQCD is obtained from NRQCD by integrating out the soft scale $m v$ [16]. Therefore, only degrees of freedom of energy $m v^{2}$ remain dynamical in pNRQCD.

${ }^{2}$ This means up to order $v^{2}$ in the Lagrangian or up to order $v^{4}$ in the spectrum. At higher orders the octet couples with the singlet through ultrasoft gluons and has to be considered [16].

${ }^{3}$ This structure already implements some of the constraints, like translational invariance, coming from the following discussion on the Poincaré group.
}

$$
\begin{aligned}
V^{(1,1)}= & -\frac{1}{2}\left\{\mathbf{p}_{1} \cdot \mathbf{p}_{2}, V_{\mathbf{p}^{2}}^{(1,1)}(r)\right\}-\frac{V_{\mathbf{L}^{2}}^{(1,1)}(r)}{2 r^{2}} \\
& \times\left(\mathbf{L}_{1} \cdot \mathbf{L}_{2}+\mathbf{L}_{2} \cdot \mathbf{L}_{1}\right)+V_{r}^{(1,1)}(r) \\
& +V_{L_{1} S_{2}}^{(1,1)}(r) \mathbf{L}_{1} \cdot \mathbf{S}_{2}-V_{L_{2} S_{1}}^{(1,1)}(r) \mathbf{L}_{2} \cdot \mathbf{S}_{1} \\
& +V_{S^{2}}^{(1,1)}(r) \mathbf{S}_{1} \cdot \mathbf{S}_{2}+V_{S_{12}}^{(1,1)}(r) \mathbf{S}_{12}(\hat{\mathbf{r}}),
\end{aligned}
$$

where $\quad \mathbf{r}=\mathbf{x}_{1}-\mathbf{x}_{2}, \quad \mathbf{L}_{j} \equiv \mathbf{r} \times \mathbf{p}_{j} \quad$ and $\quad \mathbf{S}_{12}(\hat{\mathbf{r}}) \equiv 12 \hat{\mathbf{r}} \cdot \mathbf{S}_{1} \hat{\mathbf{r}} \cdot \mathbf{S}_{2}$ $-4 S_{1} \cdot S_{2}$. The explicit form of the potentials has been determined in $[1,2]$ in terms of Wilson loop operators and will be the subject of Sec. IV.

\section{POINCARÉ INVARIANCE}

Given a specific system, the generators $\mathbf{P}$ of space translations, the generator $H$ of time translations, the generators $\mathbf{J}$ of rotations and the generators $\mathbf{K}$ of Lorentz transformations satisfy the well-known Poincaré algebra (some original references can be found in [17])

$$
\begin{aligned}
& {\left[\mathbf{P}_{i}, \mathbf{P}_{j}\right]=0,} \\
& {\left[\mathbf{P}_{i}, H\right]=0,} \\
& {\left[\mathbf{J}_{i}, \mathbf{P}_{j}\right]=i \epsilon^{i j k} \mathbf{P}_{k},} \\
& {\left[\mathbf{J}_{i}, H\right]=0,} \\
& {\left[\mathbf{J}_{i}, \mathbf{J}_{j}\right]=i \epsilon^{i j k} \mathbf{J}_{k},} \\
& {\left[\mathbf{P}_{i}, \mathbf{K}_{j}\right]=-i \delta_{i j} H,} \\
& {\left[H, \mathbf{K}_{i}\right]=-i \mathbf{P}_{i},} \\
& {\left[\mathbf{J}_{i}, \mathbf{K}_{j}\right]=i \epsilon^{i j k} \mathbf{K}_{k},} \\
& {\left[\mathbf{K}_{i}, \mathbf{K}_{j}\right]=-i \epsilon^{i j k} \mathbf{J}_{k} .}
\end{aligned}
$$

Let us consider a two-particle system of the type presented in Sec. II. We may identify the generators $\mathbf{P}$ and $\mathbf{J}$ with the total momentum and the total angular momentum of the system respectively:

$$
\begin{aligned}
& \mathbf{P}=\mathbf{p}_{1}+\mathbf{p}_{2}, \quad \mathbf{p}_{j}=-i \boldsymbol{\nabla}_{\mathbf{x}_{j}}, \\
& \mathbf{J}=\mathbf{x}_{1} \times \mathbf{p}_{1}+\mathbf{x}_{2} \times \mathbf{p}_{2}+\mathbf{S}_{1}+\mathbf{S}_{2} .
\end{aligned}
$$

These definitions satisfy Eqs. (6), (8) and (10). The meaning of the conditions set by Eqs. (7) and (9) is trivial. The first equation constrains $H$ to be a function of $\mathbf{x}_{1}-\mathbf{x}_{2}$, i.e., of the distance $\mathbf{r}$ of the two particles, only. The latter constrains $H$ to be a scalar under rotations. Of the remaining four commu- 
tators only three are independent $[18]{ }^{4}$ The constraints that they put on the form of the generators is by far less trivial and will be the main subject of this section.

We consider our two particle system to be described by the Lagrangian (1) and we identify the generator $H$ with

$$
H\left(\mathbf{x}_{1}, \mathbf{x}_{2}, \mathbf{p}_{1}, \mathbf{p}_{2}\right)=m_{1}+m_{2}+h_{s}\left(\mathbf{x}_{1}, \mathbf{x}_{2}, \mathbf{p}_{1}, \mathbf{p}_{2}\right) .
$$

It has been proved in [17] that $\mathbf{K}$ can be written as

$$
\begin{aligned}
\mathbf{K}= & \frac{1}{2} \sum_{i=1}^{2}\left[\left\{\mathbf{x}_{i}, m_{i}+\frac{\mathbf{p}_{\mathbf{i}}^{2}}{2 m_{i}}+\cdots\right\}-\frac{\mathbf{S}_{i} \times \mathbf{p}_{i}}{m_{i}}(1+\cdots)\right]-t \mathbf{P} \\
& +\mathbf{U}
\end{aligned}
$$

where $\{A, B\}=A B+B A$, and the dots indicate higher order terms in the inverse of the mass. These are given in closed form in the quoted literature, but do not matter here. ${ }^{5} \mathbf{U}$ is a function, which, due to Eq. (13), has to be a vector under rotations. Moreover, it is related to $h_{s}$ via Eq. (11). To order $1 / m$ the function $\mathbf{U}$ has to satisfy the relation

$$
\begin{aligned}
\mathbf{U}= & \mathbf{x}_{1}\left(v_{a}^{(0)}(r)+\frac{v_{a}^{(1,0)}(r)}{m_{1}}+\frac{v_{a}^{(0,1)}(r)}{m_{2}}\right) \\
& +\mathbf{x}_{2}\left(v_{b}^{(0)}(r)+\frac{v_{b}^{(1,0)}(r)}{m_{1}}+\frac{v_{b}^{(0,1)}(r)}{m_{2}}\right) \\
& +\mathbf{U}^{(0)}+\frac{\mathbf{U}^{(1,0)}}{m_{1}}+\frac{\mathbf{U}^{(0,1)}}{m_{2}}+\ldots
\end{aligned}
$$

where the functions $v_{a, b}$ have to fulfill the conditions

$$
\begin{gathered}
V^{(0)}(r)=v_{a}^{(0)}(r)+v_{b}^{(0)}(r), \\
V^{(1,0)}(r)=v_{a}^{(1,0)}(r)+v_{b}^{(1,0)}(r), \\
V^{(0,1)}(r)=v_{a}^{(0,1)}(r)+v_{b}^{(0,1)}(r) .
\end{gathered}
$$

The functions $\mathbf{U}^{(0)}, \mathbf{U}^{(1,0)}$ and $\mathbf{U}^{(0,1)}$ are arbitrary, but $\mathbf{K}$ obtained from them has to fulfill Eqs. (11)-(14). In particular, they are vectors and commute with $\mathbf{P}$, i.e., they depend on momenta and the relative distance $\mathbf{r}$ only [19]. Moreover, $\mathbf{U}^{(0)}$ commutes with $V^{(0)}$.

The relevant point is the following. $H$ is now established up to order $1 / \mathrm{m}^{2}$, Eqs. (17) and (2), and $\mathbf{K}$ up to order $1 / m$,

${ }^{4}$ For instance, by using the Jacobi identity, it can be proven that Eq. (12) follows from Eqs. (11), (13) and (14). However, we will not make explicit use of this fact in the following. The reason is that we shall analyze the Poincaré algebra in the framework of a $1 / \mathrm{m}$ expansion. In this framework, when using $H$ and $\mathbf{K}$ up to a fixed order in $1 / m$, the commutation relations are not all verified at the same order in $1 / m$ [19].

${ }^{5}$ One may wonder about the origin of the term $-t \mathbf{P}$ in Eq. (18). It is a consequence of the identification of Eq. (17) and the request of Lorentz invariance for the Lagrangian (1) (or, which is the same, for the Schrödinger equation). From the commutation relation (12) it then follows that the $t$ dependence of $\mathbf{K}$ is carried by a term $-t \mathbf{P}$.
Eqs. (18) and (19). Then Eqs. (11), (12) and (13) have to be fulfilled up to order $1 / \mathrm{m}$ and Eq. (14) up to order $1 / \mathrm{m}^{0}$ (consider that $\mathbf{K}$ starts from order $m$ ). The above definitions of $H$ and $\mathbf{K}$ trivially satisfy Eqs. (11), (13) and (14). The situation is different for Eq. (12). In order that this relation holds at order $1 / m$ some non-trivial relations between the potentials of Eq. (2) have to be fulfilled. Therefore, as already pointed out in [20], the Poincaré algebra may serve to constrain the form of the potentials. More specifically, from Eq. (12), taken up to order $1 / m$, after a lengthy calculation, we obtain relations in the spin sector of the heavy-quark potential,

$$
\begin{gathered}
V_{L S}^{(2,0)}(r)-V_{L_{2} S_{1}}^{(1,1)}(r)+\frac{1}{2 r} \frac{d V^{(0)}(r)}{d r}=0, \\
V_{L S}^{(0,2)}(r)-V_{L_{1} S_{2}}^{(1,1)}(r)+\frac{1}{2 r} \frac{d V^{(0)}(r)}{d r}=0,
\end{gathered}
$$

and in the spin-independent sector,

$$
\begin{gathered}
V_{\mathbf{L}^{2}}^{(2,0)}(r)+V_{\mathbf{L}^{2}}^{(0,2)}(r)-V_{\mathbf{L}^{2}}^{(1,1)}(r)+\frac{r}{2} \frac{d V^{(0)}(r)}{d r}=0, \\
-2\left[V_{\mathbf{p}^{2}}^{(2,0)}(r)+V_{\mathbf{p}^{2}}^{(0,2)}(r)\right]+2 V_{\mathbf{p}^{2}}^{(1,1)}(r)-V^{(0)}(r)+r \frac{d V^{(0)}(r)}{d r} \\
=0, \\
\frac{1}{r}\left[V_{\mathbf{L}^{2}}^{(2,0)}(r)-V_{\mathbf{L}^{2}}^{(0,2)}(r)\right]+\frac{d}{d r}\left[V_{\mathbf{L}^{2}}^{(2,0)}(r)-V_{\mathbf{L}^{2}}^{(0,2)}(r)\right] \\
\quad+\frac{d}{d r}\left[V_{\mathbf{p}^{2}}^{(2,0)}(r)-V_{\mathbf{p}^{2}}^{(0,2)}(r)\right]=0 .
\end{gathered}
$$

Notably the dependence on $\mathbf{U}^{(0)}, \mathbf{U}^{(1,0)}$ and $\mathbf{U}^{(0,1)}$ disappears in the final relations (for more details see Appendix A). If the system is invariant under $m_{1} \leftrightarrow m_{2}$ transformation (and considering that one of the two particles is an antiparticle), we have the additional conditions

$$
\begin{aligned}
V_{\mathbf{p}^{2}, \mathbf{L}^{2}}^{(2,0)}(r) & =V_{\mathbf{p}^{2}, \mathbf{L}^{2}}^{(0,2)}(r), \\
V_{r, L S}^{(2,0)}(r) & =V_{r, L S}^{(0,2)}\left(r ; m_{2} \leftrightarrow m_{1}\right), \\
V_{L_{1} S_{2}}^{(1,1)}(r) & =V_{L_{2} S_{1}}^{(1,1)}\left(r ; m_{1} \leftrightarrow m_{2}\right) .
\end{aligned}
$$

In this specific situation Eqs. (21) and (22) reduce, up to the NRQCD matching coefficients, to the relation obtained in [12] and Eqs. (23) -(25) to the relations obtained in [13]. In both cases the proof was done by explicit calculation on the potentials expressed in terms of Wilson loops (see Sec. IV). A proof derived from reparametrization invariance on the form of the scattering amplitude can be found in [14].

We note that constraints on the potentials $V^{(1,0)}, V^{(0,1)}$, $V_{r}, V_{S^{2}}$ and $V_{S_{12}}$ could be obtained in this way only by including terms of order $1 / \mathrm{m}^{3}$ in the Hamiltonian (2). These terms are beyond our present knowledge. 


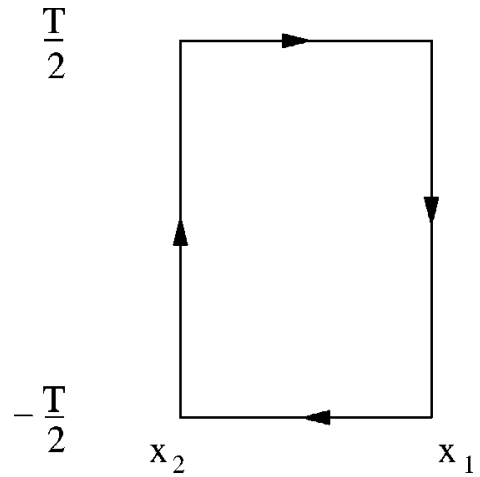

FIG. 1. Graphical representation of the static Wilson loop $W_{\square}$ of extension $r \times T\left(\mathbf{r}=\mathbf{x}_{1}-\mathbf{x}_{2}\right)$.

\section{Unitary transformations}

The Hamiltonian and hence the potentials are defined up to unitary transformations. One may wonder if this ambiguity in the definition of the potentials also affects the above exact relations. We will show, for the example of the unitary transformation considered in [1], that this is not the case and that the relations derived from Poincare invariance are invariant under unitary transformations.

In [1] the following unitary transformation has been considered:

$$
U=\exp \left(-i\left\{\mathbf{W}(\mathbf{r}), \frac{\mathbf{p}_{1}}{m_{1}}-\frac{\mathbf{p}_{2}}{m_{2}}\right\}\right),
$$

which transforms $h_{s} \rightarrow h_{s}^{\prime}=U^{\dagger} h_{s} U$. We will check explicitly that the relations $(21)-(25)$ are preserved by the transformation (29). Up to order $1 / m^{2}, h_{s}^{\prime}$ is given by

$$
\begin{aligned}
h_{s}^{\prime}= & h_{s}-\frac{1}{2 m_{1}^{2}}\left\{\mathbf{p}_{1}^{i},\left\{\mathbf{p}_{1}^{j},\left(\nabla_{r}^{i} W^{j}\right)\right\}\right\}+\frac{1}{2 m_{1} m_{2}}\left\{\mathbf{p}_{1}^{j},\left\{\mathbf{p}_{2}^{i},\left(\nabla_{r}^{i} W^{j}\right)\right\}\right\} \\
& -\frac{1}{2 m_{2}^{2}}\left\{\mathbf{p}_{2}^{i},\left\{\mathbf{p}_{2}^{j},\left(\nabla_{r}^{i} W^{j}\right)\right\}\right\}+\frac{1}{2 m_{1} m_{2}}\left\{\mathbf{p}_{1}^{i},\left\{\mathbf{p}_{2}^{j},\left(\nabla_{r}^{i} W^{j}\right)\right\}\right\} \\
& + \text { momentum independent terms. }
\end{aligned}
$$

Let us now assume a decomposition of the form $\nabla_{r}^{i} W^{j}$ $=\hat{r}^{i} \hat{r}^{j} A(r)+\delta^{i j} B(r)$. Then the momentum-dependent potentials of $h_{s}^{\prime}$ can be written as

$$
\begin{aligned}
& V_{\mathbf{L}^{2}}^{(1,1)}(r)^{\prime}=V_{\mathbf{L}^{2}}^{(1,1)}(r)+4 A(r), \\
& V_{\mathbf{L}^{2}}^{(2,0)}(r)^{\prime}=V_{\mathbf{L}^{2}}^{(2,0)}(r)+2 A(r), \\
& V_{\mathbf{L}^{2}}^{(0,2)}(r)^{\prime}=V_{\mathbf{L}^{2}}^{(0,2)}(r)+2 A(r), \\
& V_{\mathbf{p}^{2}}^{(1,1)}(r)^{\prime}=V_{\mathbf{p}^{2}}^{(1,1)}(r)-4 A(r)-4 B(r), \\
& V_{\mathbf{p}^{2}}^{(2,0)}(r)^{\prime}=V_{\mathbf{p}^{2}}^{(2,0)}(r)-2 A(r)-2 B(r), \\
& V_{\mathbf{p}^{2}}^{(0,2)}(r)^{\prime}=V_{\mathbf{p}^{2}}^{(0,2)}(r)-2 A(r)-2 B(r) .
\end{aligned}
$$

It then follows immediately that also the potentials obtained after the unitary transformation satisfy the relations (21)(25).

\section{RELATIONS OBTAINED VIA EXPLICIT TRANSFORMATIONS OF THE OPERATORS}

In this section we will prove the relations (21)-(24) under the conditions (26)-(28) by explicit transformation of the potentials,

$$
\begin{aligned}
V^{(0)}(r)= & \lim _{T \rightarrow \infty} \frac{i}{T} \ln \left\langle W_{\square}\right\rangle, \\
V_{L S}^{(2,0)}(r)= & \frac{c_{F}^{(1)}}{2 r^{2}} i \mathbf{r} \cdot \lim _{T \rightarrow \infty} \frac{1}{T} \int_{-T / 2}^{T / 2} d t \int_{-T / 2}^{T / 2} d t^{\prime \prime}\left(t-t^{\prime \prime}\right) \\
& \times\left\langle\left\langle g \mathbf{B}\left(\mathbf{x}_{1}, t^{\prime \prime}\right) \times g \mathbf{E}\left(\mathbf{x}_{1}, t\right)\right\rangle\right\rangle \\
& +\frac{c_{S}^{(1)}}{2 r^{2}} \mathbf{r} \cdot\left(\nabla_{r} V^{(0)}\right),
\end{aligned}
$$$$
V_{L_{2} S_{1}}^{(1,1)}(r)=\frac{c_{F}^{(1)}}{2 r^{2}} i \mathbf{r} \cdot \lim _{T \rightarrow \infty} \frac{1}{T} \int_{-T / 2}^{T / 2} d t \int_{-T / 2}^{T / 2} d t^{\prime \prime}\left(t-t^{\prime \prime}\right)
$$

$$
\begin{aligned}
& \times\left\langle\left\langle g \mathbf{B}\left(\mathbf{x}_{1}, t^{\prime \prime}\right) \times g \mathbf{E}\left(\mathbf{x}_{2}, t\right)\right\rangle\right\rangle \\
V_{\mathbf{p}^{2}}^{(2,0)}(r)= & \frac{i}{4} \hat{\mathbf{r}}^{i} \hat{\mathbf{r}}^{j} \lim _{T \rightarrow \infty} \frac{1}{T} \int_{-T / 2}^{T / 2} d t \int_{-T / 2}^{T / 2} d t^{\prime \prime}\left(t-t^{\prime \prime}\right)^{2} \\
& \times\left\langle\left\langle g \mathbf{E}^{i}\left(\mathbf{x}_{1}, t^{\prime \prime}\right) g \mathbf{E}^{j}\left(\mathbf{x}_{1}, t\right)\right\rangle\right\rangle_{c} \\
V_{\mathbf{L}^{2}}^{(2,0)}(r)= & i \frac{\delta^{i j}-3 \hat{\mathbf{r}}^{i} \hat{\mathbf{r}}^{j}}{8} \lim \frac{1}{T} \int_{-T / 2}^{T / 2} d t \int_{-T / 2}^{T / 2} d t^{\prime \prime}\left(t-t^{\prime \prime}\right)^{2} \\
& \times\left\langle\left\langle g \mathbf{E}^{i}\left(\mathbf{x}_{1}, t^{\prime \prime}\right) g \mathbf{E}^{j}\left(\mathbf{x}_{1}, t\right)\right\rangle\right\rangle_{c}, \\
V_{\mathbf{p}^{2}}^{(1,1)}(r)= & \frac{i}{2} \hat{\mathbf{r}}^{i} \hat{\mathbf{r}}^{j} \lim _{T \rightarrow \infty} \frac{1}{T} \int_{-T / 2}^{T / 2} d t \int_{-T / 2}^{T / 2} d t^{\prime \prime}\left(t-t^{\prime \prime}\right)^{2} \\
& \times\left\langle\left\langle g \mathbf{E}^{i}\left(\mathbf{x}_{1}, t^{\prime \prime}\right) g \mathbf{E}^{j}\left(\mathbf{x}_{2}, t\right)\right\rangle\right\rangle_{c}, \\
V_{\mathbf{L}^{2}}^{(1,1)}(r)= & i \frac{\delta^{i j}-3 \hat{\mathbf{r}}^{i} \hat{\mathbf{r}}^{j}}{4} \lim \frac{1}{T} \int_{-T / 2}^{T / 2} d t \int_{-T / 2}^{T / 2} d t^{\prime \prime}\left(t-t^{\prime \prime}\right)^{2} \\
& \times\left\langle\left\langle g \mathbf{E}^{i}\left(\mathbf{x}_{1}, t^{\prime \prime}\right) g \mathbf{E}^{j}\left(\mathbf{x}_{2}, t\right)\right\rangle\right\rangle_{c},
\end{aligned}
$$

taken from Ref. [2] (changing, for further convenience, single in double integrals). In order to ease the reader we use the same notation as [2], i.e., the angular brackets $\langle\ldots\rangle$ stand for the average value over the Yang-Mills action, $W_{\square}$ for the rectangular static Wilson loop of extension $r \times T$ (the time runs from $-T / 2$ to $T / 2$, the space coordinate from $\mathbf{x}_{1}$ to $\left.\mathbf{x}_{2}\right)$ :

$$
W_{\square} \equiv \mathrm{P} \exp \left\{-i g \oint_{r \times T} d z^{\mu} A_{\mu}(z)\right\},
$$




$$
d z^{\mu} A_{\mu} \equiv d z^{0} A_{0}-d \mathbf{z} \cdot \mathbf{A},
$$

which we graphically represent in Fig. 1, and $\langle\langle\ldots\rangle\rangle$ $\equiv\left\langle\ldots W_{\square}\right\rangle /\left\langle W_{\square}\right\rangle ; \mathrm{P}$ is the path-ordering operator. Moreover, we define the connected Wilson loop with $O_{1}\left(t_{1}\right), O_{2}\left(t_{2}\right)$ and $\mathrm{O}_{3}\left(t_{3}\right)$ operator insertions by:

$$
\begin{aligned}
&\left\langle\left\langle O_{1}\left(t_{1}\right) O_{2}\left(t_{2}\right) O_{3}\left(t_{3}\right)\right\rangle\right\rangle_{c} \\
&=\left\langle\left\langle O_{1}\left(t_{1}\right) O_{2}\left(t_{2}\right) O_{3}\left(t_{3}\right)\right\rangle\right\rangle-\left\langle\left\langle O_{1}\left(t_{1}\right)\right\rangle\right\rangle\left\langle\left\langle O_{2}\left(t_{2}\right)\right\rangle\right\rangle \\
& \times\left\langle\left\langle O_{3}\left(t_{3}\right)\right\rangle\right\rangle-\left\langle\left\langle O_{1}\left(t_{1}\right)\right\rangle\right\rangle\left\langle\left\langle O_{2}\left(t_{2}\right) O_{3}\left(t_{3}\right)\right\rangle\right\rangle_{c} \\
&-\left\langle\left\langle O_{2}\left(t_{2}\right)\right\rangle\right\rangle\left\langle\left\langle O_{1}\left(t_{1}\right) O_{3}\left(t_{3}\right)\right\rangle\right\rangle_{c}-\left\langle\left\langle O_{3}\left(t_{3}\right)\right\rangle\right\rangle \\
& \times\left\langle\left\langle O_{1}\left(t_{1}\right) O_{2}\left(t_{2}\right)\right\rangle\right\rangle_{c}, \\
&\left\langle\left\langle O_{1}\left(t_{1}\right) O_{2}\left(t_{2}\right)\right\rangle\right\rangle_{c} \\
&=\left\langle\left\langle O_{1}\left(t_{1}\right) O_{2}\left(t_{2}\right)\right\rangle\right\rangle-\left\langle\left\langle O_{1}\left(t_{1}\right)\right\rangle\right\rangle\left\langle\left\langle O_{2}\left(t_{2}\right)\right\rangle\right\rangle .
\end{aligned}
$$

The operators $\mathbf{E}^{i}=F_{0 i}$ and $\mathbf{B}^{i}=\epsilon^{i j k} F^{j k} / 2 \quad\left(F_{\mu \nu}=\partial_{\mu} A_{\nu}\right.$ $\left.-\partial_{\nu} A_{\mu}+i g\left[A_{\mu}, A_{\nu}\right]\right)$ are the chromoelectric and chromomagnetic field respectively.

The matching coefficients $c_{F}^{(j)}, c_{S}^{(j)}$ are inherited from the matching between QCD and NRQCD. Their explicit form does not matter here. The only point relevant for our further discussion is that, from reparametrization invariance, one has [21]

$$
c_{S}^{(j)}=2 c_{F}^{(j)}-1
$$

\section{A. Relations for spin-dependent potentials}

In this section we derive the relations (21) and (22) by explicit transformation of the potentials (32) and (33). The proof is, partially, a simplified version of that derived long ago by one of the authors $[12,6]$.

We start from the identity

$$
\left\langle\left\langle g \mathbf{B}\left(\mathbf{x}_{1}, t^{\prime \prime}\right)\right\rangle\right\rangle=0,
$$

which follows from parity. Then we apply an infinitesimal Lorentz boost with velocity $\mathbf{v}$, and the point $\left(\mathbf{x}_{1}, t^{\prime \prime}\right)$ as the origin in both systems, i.e., up to order $\mathcal{O}(\mathrm{v})$

$$
\begin{gathered}
\mathbf{x}-\mathbf{x}_{1}=\mathbf{x}^{\prime}-\mathbf{x}_{1}+\left(t^{\prime}-t^{\prime \prime}\right) \mathbf{v} \\
t-t^{\prime \prime}=t^{\prime}-t^{\prime \prime}+\left(\mathbf{x}^{\prime}-\mathbf{x}_{1}^{\prime}\right) \cdot \mathbf{v}
\end{gathered}
$$

The Wilson loop transforms like in Fig. 2. The time-like paths become $\mathbf{x}_{1,2}^{\prime}\left(t^{\prime}\right)=\mathbf{x}_{1,2}-\left(t^{\prime}-t^{\prime \prime}\right) \mathbf{v}$, with the boundaries $t_{ \pm}^{(1,2)^{\prime}}= \pm T / 2-\left(\mathbf{x}_{1,2}-\mathbf{x}_{1}\right) \mathbf{v}$. To make the last contribution vanish it is convenient to choose $\mathbf{v} \perp \mathbf{r}$ in the remainder of this section. The spatial lines become $\mathbf{x}_{ \pm}^{\prime}(s)=\mathbf{x}_{2}+s\left(\mathbf{x}_{1}-\mathbf{x}_{2}\right)$ $-\mathbf{v}\left( \pm T / 2-t^{\prime \prime}\right), 0 \leqslant s \leqslant 1$. In the new system Eq. (40) becomes

$$
\left\langle\left\langle g \mathbf{B}^{\prime}\left(\mathbf{x}_{1}, t^{\prime \prime}\right)\right\rangle\right\rangle^{\prime}+\left\langle\left\langle\left[\mathbf{v} \times g \mathbf{E}^{\prime}\left(\mathbf{x}_{1}, t^{\prime \prime}\right)\right]\right\rangle\right\rangle^{\prime}=0 .
$$

The second term comes from the transformation of the magnetic field, and the prime on the double brackets denotes that we have to use the transformed Wilson loop. We next drop

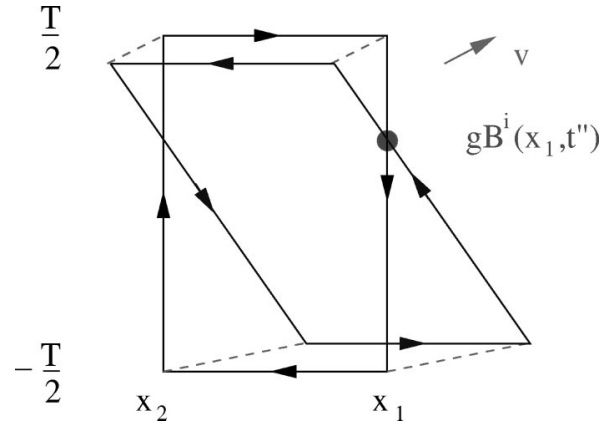

FIG. 2. Difference between the boosted expression (40) and the original one. The arrows indicate the orientation of the loops, which arise after expanding up to first order of $\mathrm{v}$. The corners of the old and the new loop have been connected by dashed lines, in order to visualize the form of the infinitesimal loops, which correspond to the insertions of chromoelectric (time-like triangles at $\mathbf{x}_{1}$ and $\mathbf{x}_{2}$ ) and chromomagnetic fields (space-like parallelograms at $\pm T / 2$ ).

the primes for the fields and consider Eq. (42) in the old system. Subtracting Eq. (40) from Eq. (42), dividing by $T$, and integrating over $t^{\prime \prime}$ we get at order $\mathcal{O}(\mathrm{v})$

$$
\begin{gathered}
\frac{1}{T} \int_{-T / 2}^{T / 2} d t^{\prime \prime}\left\langle\left\langle g \mathbf{B}\left(\mathbf{x}_{1}, t^{\prime \prime}\right)\right\rangle\right\rangle^{\prime}-\frac{1}{T} \int_{-T / 2}^{T / 2} d t^{\prime \prime}\left\langle\left\langle g \mathbf{B}\left(\mathbf{x}_{1}, t^{\prime \prime}\right)\right\rangle\right\rangle \\
+\frac{1}{T} \int_{-T / 2}^{T / 2} d t^{\prime \prime}\left\langle\left\langle\left[\mathbf{v} \times g \mathbf{E}\left(\mathbf{x}_{1}, t^{\prime \prime}\right)\right]\right\rangle\right\rangle=0
\end{gathered}
$$

Notice that the second term of Eq. (42) has been just rotated back because it is already of order $\mathcal{O}(\mathrm{v})$. Rewriting the differences in the paths of the first and second term in Eq. (43) as insertions of chromoelectric and chromomagnetic fields, we get, up to order $\mathcal{O}(\mathrm{v})$

$$
\begin{aligned}
\mathbf{v}^{i}\left\{\frac { i } { T } \int _ { - T / 2 } ^ { T / 2 } d t ^ { \prime \prime } \int _ { - T / 2 } ^ { T / 2 } d t ( t - t ^ { \prime \prime } ) \left[\left\langle\left\langle g \mathbf{B}^{k}\left(\mathbf{x}_{1}, t^{\prime \prime}\right) g \mathbf{E}^{i}\left(\mathbf{x}_{1}, t\right)\right\rangle\right\rangle\right.\right. \\
\left.-\left\langle\left\langle g \mathbf{B}^{k}\left(\mathbf{x}_{1}, t^{\prime \prime}\right) g \mathbf{E}^{i}\left(\mathbf{x}_{2}, t\right)\right\rangle\right\rangle\right]+\frac{i}{T} \epsilon^{i j l} \mathbf{r}^{j} \int_{0}^{1} d s \int_{-T / 2}^{T / 2} d t^{\prime \prime} \\
\quad \times\left[\left(t^{\prime \prime}+\frac{T}{2}\right)\left\langle\left\langle g \mathbf{B}^{k}\left(\mathbf{x}_{1}, t^{\prime \prime}\right) g \mathbf{B}^{l}\left(\mathbf{x}_{2}+s \mathbf{r},-T / 2\right)\right\rangle\right\rangle\right. \\
\left.-\left(t^{\prime \prime}-\frac{T}{2}\right)\left\langle\left\langle g \mathbf{B}^{l}\left(\mathbf{x}_{2}+s \mathbf{r}, T / 2\right) g \mathbf{B}^{k}\left(\mathbf{x}_{1}, t^{\prime \prime}\right)\right\rangle\right\rangle\right] \\
\left.+\frac{1}{T} \epsilon^{k i j} \int_{-T / 2}^{T / 2} d t^{\prime \prime}\left\langle\left\langle g \mathbf{E}^{j}\left(\mathbf{x}_{1}, t^{\prime \prime}\right)\right\rangle\right\rangle\right\}=0
\end{aligned}
$$

Now we consider the large $T$ limit of Eq. (44). In [1] it has been proved that, for $T \rightarrow \infty$,

$$
\frac{1}{T} \int_{-T / 2}^{T / 2} d t^{\prime \prime}\left\langle\left\langle g \mathbf{E}^{j}\left(\mathbf{x}_{1}, t^{\prime \prime}\right)\right\rangle\right\rangle=\frac{\mathbf{r}^{j}}{r} \frac{d}{d r} V^{(0)}(r)+\mathcal{O}\left(\frac{1}{T}\right)
$$

Moreover, we have for $T \rightarrow \infty$ 


$$
\begin{gathered}
\frac{i}{T} \int_{-T / 2}^{T / 2} d t^{\prime \prime}\left[\left(t^{\prime \prime}+\frac{T}{2}\right)\left\langle\left\langle g \mathbf{B}^{k}\left(\mathbf{x}_{1}, t^{\prime \prime}\right) g \mathbf{B}^{l}\left(\mathbf{x}_{2}+s \mathbf{r},-T / 2\right)\right\rangle\right\rangle\right. \\
\left.-\left(t^{\prime \prime}-\frac{T}{2}\right)\left\langle\left\langle g \mathbf{B}^{l}\left(\mathbf{x}_{2}+s \mathbf{r}, T / 2\right) g \mathbf{B}^{k}\left(\mathbf{x}_{1}, t^{\prime \prime}\right)\right\rangle\right\rangle\right] \\
=2 \sum_{n \neq 0} \frac{a_{n} \mathbf{b}_{0}^{l}}{a_{0}^{2}} \frac{{ }^{(0)}\left\langle n\left|g \mathbf{B}^{k}\left(\mathbf{x}_{1}\right)\right| 0\right\rangle^{(0)}}{E_{n}^{(0)}-E_{0}^{(0)}}+\mathcal{O}\left(\frac{1}{T}\right)
\end{gathered}
$$

where $|n\rangle^{(0)}$ are the eigenstates of the static NRQCD Hamiltonian, $a_{n}$ is the projection on $|n\rangle^{(0)}$ of the state made by two static quarks connected by a straight string, and $\mathbf{b}_{n}^{l}$ of the state made by two static quarks and the chromomagnetic field $g \mathbf{B}^{l}\left(\mathbf{x}_{2}+s \mathbf{r},-T / 2\right)$ connected by a straight string (for more specification on this way of analyzing the large $T$ limit of Wilson loops we refer to $[1,2])$. Since, due to the different quantum numbers, this latter state has no overlap with the ground state $|0\rangle^{(0)}$, which we identify with the color singlet quarkonium state $S$ introduced in Sec. II, we have $\mathbf{b}_{0}^{l}=0$. Therefore, the left-hand side of Eq. (46), vanishes in the large $T$ limit.

From the above analysis it follows that Eq. (44) reduces, in the large $T$ limit, to the identity

$$
\begin{aligned}
\lim _{T \rightarrow \infty} \frac{i}{T} \int_{-T / 2}^{T / 2} d t^{\prime \prime} \int_{-T / 2}^{T / 2} d t\left(t-t^{\prime \prime}\right)\left[\left\langle\left\langle g \mathbf{B}\left(\mathbf{x}_{1}, t^{\prime \prime}\right) \times g \mathbf{E}\left(\mathbf{x}_{1}, t\right)\right\rangle\right\rangle\right. \\
\left.-\left\langle\left\langle g \mathbf{B}\left(\mathbf{x}_{1}, t^{\prime \prime}\right) \times g \mathbf{E}\left(\mathbf{x}_{2}, t\right)\right\rangle\right\rangle\right]+2 \frac{\mathbf{r}}{r} \frac{d}{d r} V^{(0)}(r)=0 .
\end{aligned}
$$

This corresponds to the relation ${ }^{6} V_{2}^{\prime}-V_{1}^{\prime}=V^{(0)^{\prime}}$, given first in Ref. [12]. Moreover, using Eq. (39), we obtain Eqs. (21) and (22).

We would like to remark that this result depends crucially on the fact that the contributions from the shifts of the spacelike lines of the Wilson loop in Eq. (46) vanish in the large $T$ limit. These contributions were apparently never considered in the literature so far and it may be regarded as a largely lucky circumstance that they actually do not affect the iden-

\footnotetext{
${ }^{6}$ The potentials $V_{1}^{\prime}, V_{2}^{\prime}$, which, up to missing Wilson coefficients not considered in the early literature, correspond to the spin-orbit potentials, are defined as

$$
\begin{aligned}
V_{1}^{\prime}(r)= & \frac{i}{2} \frac{\mathbf{r}}{r} \cdot \lim _{T \rightarrow \infty} \frac{1}{T} \int_{-T / 2}^{T / 2} d t \int_{-T / 2}^{T / 2} d t^{\prime \prime}\left(t-t^{\prime \prime}\right)\left\langle\left\langle g \mathbf{B}\left(\mathbf{x}_{1}, t^{\prime \prime}\right)\right.\right. \\
& \left.\left.\times g \mathbf{E}\left(\mathbf{x}_{1}, t\right)\right\rangle\right\rangle, \\
V_{2}^{\prime}(r)= & \frac{i}{2} \frac{\mathbf{r}}{r} \cdot \lim _{T \rightarrow \infty} \frac{1}{T} \int_{-T / 2}^{T / 2} d t \int_{-T / 2}^{T / 2} d t^{\prime \prime}\left(t-t^{\prime \prime}\right)\left\langle\left\langle g \mathbf{B}\left(\mathbf{x}_{1}, t^{\prime \prime}\right)\right.\right. \\
& \left.\left.\times g \mathbf{E}\left(\mathbf{x}_{2}, t\right)\right\rangle\right\rangle .
\end{aligned}
$$
}

See also Appendix B. tities involving the spin-dependent potentials. From a more technical point of view, this is related to the fact that the Wilson loop operators on the left-hand side of Eq. (46) are multiplied by the factors $\left(t^{\prime \prime}+T / 2\right)$ and $\left(t^{\prime \prime}-T / 2\right)$ respectively, which are identical to the time differences of the correlation functions. In different situations the corresponding end-point contributions cannot be neglected. This fact was generally overlooked in the past literature and led to wrong statements and contradictions. This will be clarified in Appendix B.

\section{B. Relations for spin-independent potentials}

In this section we derive the relations (23) and (24) by explicit computation on the potentials (34) and (37). It is convenient, for our purposes, to introduce the following definitions $^{7}[11]$ :

$$
\begin{aligned}
S^{j k}= & \delta^{j k} V_{b}+\left(\frac{\delta^{j k}}{3}-\frac{\mathbf{r}^{j} \mathbf{r}^{k}}{r^{2}}\right) V_{c} \\
= & -\frac{i}{2} \lim _{T \rightarrow \infty} \frac{1}{T} \int_{-T / 2}^{T / 2} d t^{\prime} \int_{-T / 2}^{T / 2} d t^{\prime \prime}\left(t^{\prime}-t^{\prime \prime}\right)^{2} \\
& \times\left\langle\left\langle g \mathbf{E}^{j}\left(\mathbf{x}_{2}, t^{\prime}\right) g \mathbf{E}^{k}\left(\mathbf{x}_{1}, t^{\prime \prime}\right)\right\rangle\right\rangle_{c}, \\
T^{j k}= & \delta^{j k} V_{d}+\left(\frac{\delta^{j k}}{3}-\frac{\mathbf{r}^{j} \mathbf{r}^{k}}{r^{2}}\right) V_{e} \\
= & \frac{i}{4} \lim _{T \rightarrow \infty} \frac{1}{T} \int_{-T / 2}^{T / 2} d t^{\prime} \int_{-T / 2}^{T / 2} d t^{\prime \prime}\left(t^{\prime}-t^{\prime \prime}\right)^{2} \\
& \times\left\langle\left\langle g \mathbf{E}^{j}\left(\mathbf{x}_{1}, t^{\prime}\right) g \mathbf{E}^{k}\left(\mathbf{x}_{1}, t^{\prime \prime}\right)\right\rangle\right\rangle_{c} .
\end{aligned}
$$

The above formulas involve insertions of two chromoelectric fields, together with quadratic factors in the time differences. Therefore, we have to consider the Lorentz transformation up to second order in the velocity $\mathrm{v}$.

We start with an expression like Eq. (40), but with a chromoelectric field instead of the chromomagnetic one, i.e.,

$$
\left\langle\left\langle i g \mathbf{v} \cdot \mathbf{E}\left(\mathbf{x}_{1}, t^{\prime \prime}\right)\right\rangle\right\rangle \text {. }
$$

The velocity $\mathbf{v}$ will be used in the Lorentz boost. The longitudinal component of the chromoelectric field which appears in Eq. (50) is not changed under this transformation, which greatly simplifies the derivation. Another reason for taking the scalar product with $\mathbf{v}$ will become obvious below. The Lorentz transformation with $\left(\mathbf{x}_{1}, t^{\prime \prime}\right)$ as origin is now needed up to order $\mathcal{O}\left(\mathrm{v}^{2}\right)$. It reads

$$
\begin{aligned}
& { }^{7} \text { In terms of the potentials defined at the beginning of Sec. IV, we } \\
& \text { have } \\
& \qquad V_{\mathbf{p}^{2}}^{(2,0)}=V_{d}-\frac{2}{3} V_{e}, \quad V_{\mathbf{L}^{2}}^{(2,0)}=V_{e}, \quad V_{\mathbf{p}^{2}}^{(1,1)}=-V_{b}+\frac{2}{3} V_{c}, \\
& V_{\mathbf{L}^{2}}^{(1,1)}=-V_{c} .
\end{aligned}
$$




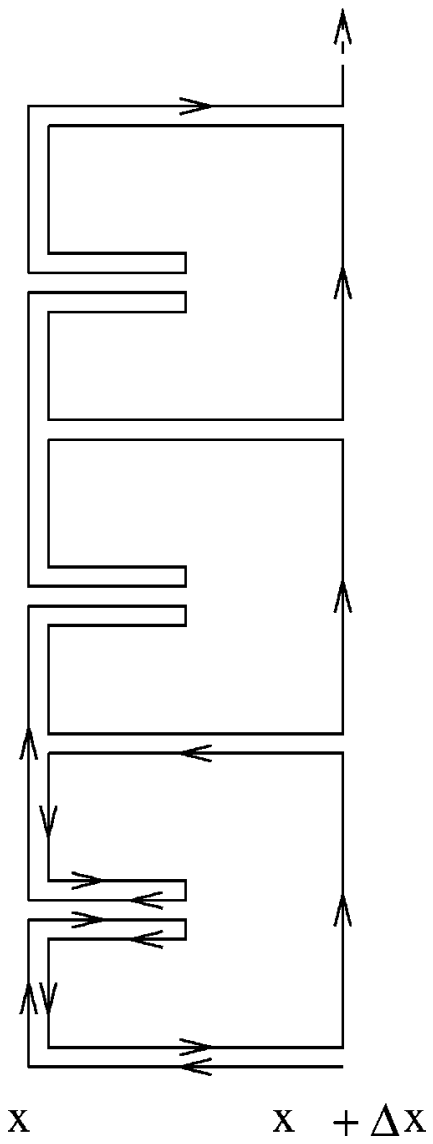

FIG. 3. Distortion of the path showing the appearance of the field insertions and the covariant derivatives.

$$
\begin{aligned}
& \mathbf{x}-\mathbf{x}_{1}=\mathbf{x}^{\prime}-\mathbf{x}_{1}+\left(t^{\prime}-t^{\prime \prime}\right) \mathbf{v}+\frac{1}{2}\left[\left(\mathbf{x}^{\prime}-\mathbf{x}_{1}\right) \cdot \mathbf{v}\right] \mathbf{v}, \\
& t-t^{\prime \prime}=\left(1+\frac{v^{2}}{2}\right)\left(t^{\prime}-t^{\prime \prime}\right)+\left(\mathbf{x}^{\prime}-\mathbf{x}_{1}\right) \cdot \mathbf{v}
\end{aligned}
$$

In the new system the time-like lines of the Wilson loop become

$$
\mathbf{x}_{1,2}^{\prime}\left(t^{\prime}\right)=\mathbf{x}_{1,2}-\left(t^{\prime}-t^{\prime \prime}\right) \mathbf{v}-\frac{1}{2}\left[\left(\mathbf{x}_{1,2}-\mathbf{x}_{1}\right) \cdot \mathbf{v}\right] \mathbf{v}
$$

with the boundaries

$$
t_{ \pm}^{(1,2)^{\prime}}= \pm\left(1+\frac{v^{2}}{2}\right) \frac{T}{2}-\left(\mathbf{x}_{1,2}-\mathbf{x}_{1}\right) \cdot \mathbf{v}-\frac{v^{2}}{2} t^{\prime \prime}
$$

The spatial lines become

$$
\begin{aligned}
\mathbf{x}_{ \pm}^{\prime}(s)= & \mathbf{x}_{2}+s\left(\mathbf{x}_{1}-\mathbf{x}_{2}\right)-\mathbf{v}\left( \pm T / 2-t^{\prime \prime}\right) \\
& +\frac{1}{2}(1-s)\left[\left(\mathbf{x}_{1}-\mathbf{x}_{2}\right) \cdot \mathbf{v}\right] \mathbf{v}, \quad 0 \leqslant s \leqslant 1 .
\end{aligned}
$$

We now write Eq. (50) in the new coordinate system. Recalling that the longitudinal component of $\mathbf{E}$ is not transformed, we obtain

$$
\left\langle\left\langle i g \mathbf{v} \cdot \mathbf{E}^{\prime}\left(\mathbf{x}_{1}, t^{\prime \prime}\right)\right\rangle\right\rangle^{\prime} .
$$

Again the prime denotes the transformed Wilson loop. We next drop the primes for the field in the boosted system and consider the difference between the expressions (55) and (50), divide by $T$, and integrate over $t^{\prime \prime}$ from $-T / 2$ to $T / 2$. This has to vanish by Lorentz invariance:

$$
\begin{aligned}
& \frac{1}{T} \int_{-T / 2}^{T / 2} d t^{\prime \prime}\left\langle\left\langle i g \mathbf{v} \cdot \mathbf{E}\left(\mathbf{x}_{1}, t^{\prime \prime}\right)\right\rangle\right\rangle^{\prime}-\frac{1}{T} \int_{-T / 2}^{T / 2} d t^{\prime \prime}\left\langle\left\langle i g \mathbf{v} \cdot \mathbf{E}\left(\mathbf{x}_{1}, t^{\prime \prime}\right)\right\rangle\right\rangle \\
& \quad=0
\end{aligned}
$$

The figure looks like Fig. 2 with $\mathbf{B}\left(\mathbf{x}_{1}, t^{\prime \prime}\right)$ replaced by $\mathbf{E}\left(\mathbf{x}_{1}, t^{\prime \prime}\right)$, but the shift of the Wilson loop lines has now to be considered up to order $\mathrm{v}^{2}$.

In the Abelian case the evaluation of the left-hand side of Eq. (56) could be easily done according to the selfexplaining scheme

$$
\begin{aligned}
\exp \left\{-i g \int_{C^{\prime}}\right\}= & \exp \left\{-i g \int_{C}-i g \int_{C^{\prime}-C}\right\} \\
= & {\left[\exp \left\{-i g \int_{C}\right\}\right] } \\
& \times\left[1-i g \int_{C^{\prime}-C}-\frac{g^{2}}{2}\left(\int_{C^{\prime}-C}\right)^{2}+\ldots\right],
\end{aligned}
$$

where $C$ is the original Wilson loop and $C^{\prime}$ the boosted one. More specifically, considering the time-like paths only, we get $\int_{C^{\prime}-C}=\int \mathbf{E} d \mathbf{a}$, where the integral is now over the area enclosed by the time-like lines of the paths $C^{\prime}$ and $C$. The two paths differ by order $\mathrm{v}$. In order to get the correct result up to order $\mathrm{v}^{2}$ one has to expand $\mathbf{E}\left(\mathbf{x}_{1,2}-s\left(t^{\prime}-t^{\prime \prime}\right) \mathbf{v}\right.$ $\left.-s\left[\mathbf{v} \cdot\left(\mathbf{x}_{1,2}-\mathbf{x}_{1}\right)\right] \mathbf{v} / 2, t^{\prime}\right)=\mathbf{E}\left(\mathbf{x}_{1,2}, t^{\prime}\right)-s\left(t^{\prime}-t^{\prime \prime}\right)(\mathbf{v} \cdot \boldsymbol{\nabla}) \mathbf{E}\left(\mathbf{x}_{1,2}, t^{\prime}\right)$ $+\mathcal{O}\left(\mathrm{v}^{2}\right)$. Here $s$ parametrizes the transversal direction between the lines $C^{\prime}$ and $C$; it has to be integrated from 0 to 1 , which gives a factor $1 / 2$ in the second term. Analogously we may calculate the contributions coming from the shifts in the spatial lines (these, however, involve also chromomagnetic fields).

In the non-Abelian case one can apply a method similar to that used in the derivation of the non-Abelian Stokes theorem [22]. Distort the line at, say, $\mathbf{x}+\Delta \mathbf{x}$ by adding appendices as shown in Fig. 3. This gives a path at $\mathbf{x}$, together with insertions of chromoelectric fields, represented by the paths around the small rectangles. They start and end in the middle of the rectangles and give the chromoelectric field at the position $\mathbf{x}+\Delta \mathbf{x} / 2$. Together with the small lines connecting the midpoint with the line at $\mathbf{x}$ one has

$$
\begin{aligned}
e^{-i g \mathbf{A}(\mathbf{x}) \cdot \Delta \mathbf{x} / 2} \mathbf{E}^{k}\left(\mathbf{x}+\frac{\Delta \mathbf{x}}{2}\right) e^{i g \mathbf{A}(\mathbf{x}) \cdot \Delta \mathbf{x} / 2} \\
=\mathbf{E}^{k}(\mathbf{x})+\frac{\Delta \mathbf{x}}{2} \cdot\left\{\left[\boldsymbol{\nabla}_{\mathbf{x}} \mathbf{E}^{k}(\mathbf{x})\right]-i g\left[\mathbf{A}(\mathbf{x}), \mathbf{E}^{k}(\mathbf{x})\right]\right\}+\ldots \\
=\mathbf{E}^{k}(\mathbf{x})+\frac{\Delta \mathbf{x}}{2} \cdot\left[\mathbf{D}_{\mathbf{x}}, \mathbf{E}^{k}(\mathbf{x})\right]+\ldots
\end{aligned}
$$


with the covariant derivative $\mathbf{D}_{\mathbf{x}}=\nabla_{\mathbf{x}}-i g \mathbf{A}(\mathbf{x})$.

Applying the above considerations to the left-hand side of Eq. (56) one finds that the contributions of order $v^{2}$ vanish by time reversal invariance, because the integrals contain factors $\left(t^{\prime}-t^{\prime \prime}\right)$, which are linear in the time variables. The spatial shifts do not contribute in the limit $T \rightarrow \infty$. This can be seen explicitly by using the same methods and arguments of Sec. IV A. The remaining contributions of order $\mathrm{v}^{3}$ are, in the large $T$ limit,

$$
\begin{aligned}
E^{(1,2)}= & \pm \lim _{T \rightarrow \infty} \frac{1}{2 T} \int_{-T / 2}^{T / 2} d t^{\prime} \int_{-T / 2}^{T / 2} d t^{\prime \prime}\left(t^{\prime}-t^{\prime \prime}\right)^{2} \\
& \times\left\langle\left\langle\left[\mathbf{v} \cdot \mathbf{D}_{\mathbf{x}_{1,2}}, g \mathbf{v} \cdot \mathbf{E}\left(\mathbf{x}_{1,2}, t^{\prime}\right)\right] g \mathbf{v} \cdot \mathbf{E}\left(\mathbf{x}_{1}, t^{\prime \prime}\right)\right\rangle\right\rangle_{c}, \\
E^{(3)}= & -\lim _{T \rightarrow \infty} \frac{1}{2 T}(\mathbf{r} \cdot \mathbf{v}) \int_{-T / 2}^{T / 2} d t^{\prime} \int_{-T / 2}^{T / 2} d t^{\prime \prime} \\
& \times\left\langle\left\langle g \mathbf{v} \cdot \mathbf{E}\left(\mathbf{x}_{2}, t^{\prime}\right) g \mathbf{v} \cdot \mathbf{E}\left(\mathbf{x}_{1}, t^{\prime \prime}\right)\right\rangle\right\rangle_{c} \\
E^{(4)}= & E_{11}^{(4)}+E_{22}^{(4)}-2 E_{12}^{(4)}
\end{aligned}
$$

with

$$
\begin{aligned}
E_{j k}^{(4)}= & -\lim _{T \rightarrow \infty} \frac{i}{2 T} \int_{-T / 2}^{T / 2} d t^{\prime} \int_{-T / 2}^{T / 2} d t^{\prime \prime} \int_{-T / 2}^{T / 2} d t^{\prime \prime \prime}\left(t^{\prime}-t^{\prime \prime}\right)\left(t^{\prime \prime \prime}-t^{\prime \prime}\right) \\
& \times\left\langle\left\langle g \mathbf{v} \cdot \mathbf{E}\left(\mathbf{x}_{j}, t^{\prime}\right) g \mathbf{v} \cdot \mathbf{E}\left(\mathbf{x}_{k}, t^{\prime \prime \prime}\right) g \mathbf{v} \cdot \mathbf{E}\left(\mathbf{x}_{1}, t^{\prime \prime}\right)\right\rangle\right\rangle_{c} .
\end{aligned}
$$

The terms $E^{(1,2)}$ arise from the first order shift (with respect to $\mathbf{v})$ of the paths, together with the first order expansion of the chromoelectric field. $E^{(3)}$ belongs to the second order shift in Eq. (52) at the line $\mathbf{x}_{2}$, while $E^{(4)}$ is due to the square of the integral in Eq. (57).

We next rewrite the commutator of the covariant derivative in $E^{(1,2)}$. This is rather simple for $E^{(2)}$ : the loop with the insertion of $\left[\mathbf{D}_{\mathbf{x}_{2}}^{j}, \mathbf{E}^{k}\left(\mathbf{x}_{2}, t^{\prime}\right)\right]$ may be written as $\boldsymbol{\nabla}_{\mathbf{x}_{2}}^{j}$ acting on the loop with the insertion of $\mathbf{E}^{k}\left(\mathbf{x}_{2}, t^{\prime}\right)$, minus $\boldsymbol{\nabla}_{\mathbf{x}_{2}}^{j}$ acting on the loop alone. The last derivative can, in turn, be expressed, in the large $T$ limit, by an additional insertion of a chromoelectric field $i g \mathbf{E}^{j}\left(\mathbf{x}_{2}, t^{\prime \prime \prime}\right)$ and integration over $t^{\prime \prime \prime}$. In this way $E^{(2)}$ becomes a sum of a derivative of a correlator with two fields and of a correlator with three fields, $E^{(2)}=E_{2}^{(2)}$ $+E_{3}^{(2)}$, with

$$
\begin{aligned}
E_{2}^{(2)}= & -\lim _{T \rightarrow \infty} \frac{1}{2 T}\left(\mathbf{v} \cdot \nabla_{\mathbf{x}_{2}}\right) \int_{-T / 2}^{T / 2} d t^{\prime} \int_{-T / 2}^{T / 2} d t^{\prime \prime}\left(t^{\prime}-t^{\prime \prime}\right)^{2} \\
& \times\left\langle\left\langle g \mathbf{v} \cdot \mathbf{E}\left(\mathbf{x}_{2}, t^{\prime}\right) g \mathbf{v} \cdot \mathbf{E}\left(\mathbf{x}_{1}, t^{\prime \prime}\right)\right\rangle\right\rangle_{c},
\end{aligned}
$$

$$
\begin{aligned}
E_{3}^{(2)}= & \lim _{T \rightarrow \infty} \frac{i}{2 T} \int_{-T / 2}^{T / 2} d t^{\prime} \int_{-T / 2}^{T / 2} d t^{\prime \prime} \int_{-T / 2}^{T / 2} d t^{\prime \prime \prime}\left(t^{\prime}-t^{\prime \prime}\right)^{2} \\
& \times\left\langle\left\langle g \mathbf{v} \cdot \mathbf{E}\left(\mathbf{x}_{2}, t^{\prime}\right) g \mathbf{v} \cdot \mathbf{E}\left(\mathbf{x}_{2}, t^{\prime \prime \prime}\right) g \mathbf{v} \cdot \mathbf{E}\left(\mathbf{x}_{1}, t^{\prime \prime}\right)\right\rangle\right\rangle_{c} .
\end{aligned}
$$

For $E^{(1)}$ the situation is slightly different, because $\boldsymbol{\nabla}_{\mathbf{x}_{1}}^{j}$ acting on the whole loop together with the insertions also differentiates $\mathbf{E}\left(\mathbf{x}_{1}, t^{\prime \prime}\right)$. The fields $\mathbf{v} \cdot \mathbf{E}\left(\mathbf{x}_{1}, t^{\prime}\right)$ and $\mathbf{v} \cdot \mathbf{E}\left(\mathbf{x}_{1}, t^{\prime \prime}\right)$ enter symmetrically, therefore differentiation gives twice the same expression. Instead of Eq. (63) we thus obtain $E^{(1)}=E_{2}^{(1)}$ $+E_{3}^{(1)}$, with

$$
\begin{aligned}
E_{2}^{(1)}= & \lim _{T \rightarrow \infty} \frac{1}{4 T}\left(\mathbf{v} \cdot \nabla_{\mathbf{x}_{1}}\right) \int_{-T / 2}^{T / 2} d t^{\prime} \int_{-T / 2}^{T / 2} d t^{\prime \prime}\left(t^{\prime}-t^{\prime \prime}\right)^{2} \\
& \times\left\langle\left\langle g \mathbf{v} \cdot \mathbf{E}\left(\mathbf{x}_{1}, t^{\prime}\right) g \mathbf{v} \cdot \mathbf{E}\left(\mathbf{x}_{1}, t^{\prime \prime}\right)\right\rangle\right\rangle_{c}, \\
E_{3}^{(1)}= & \lim _{T \rightarrow \infty} \frac{i}{4 T} \int_{-T / 2}^{T / 2} d t^{\prime} \int_{-T / 2}^{T / 2} d t^{\prime \prime} \int_{-T / 2}^{T / 2} d t^{\prime \prime \prime}\left(t^{\prime}-t^{\prime \prime}\right)^{2} \\
& \times\left\langle\left\langle g \mathbf{v} \cdot \mathbf{E}\left(\mathbf{x}_{1}, t^{\prime}\right) g \mathbf{v} \cdot \mathbf{E}\left(\mathbf{x}_{1}, t^{\prime \prime \prime}\right) g \mathbf{v} \cdot \mathbf{E}\left(\mathbf{x}_{1}, t^{\prime \prime}\right)\right\rangle\right\rangle_{c} .
\end{aligned}
$$

The correlators $E_{2}^{(2)}$ and $E_{2}^{(1)}$ can be directly written in terms of the expressions $S^{j k}$ and $T^{j k}$ in Eqs. (48) and (49). As an intermediate result we obtain

$$
E_{2}^{(2)}+E_{2}^{(1)}=i\left(\mathbf{v} \cdot \nabla_{r}\right) \mathbf{v}^{j}\left(S^{j k}-T^{j k}\right) \mathbf{v}^{k} .
$$

The contribution $E^{(3)}$ can be expressed by the static potential $V^{(0)}$ if we rewrite the insertions of the chromoelectric fields as derivatives acting on the loop. It can be written in the form

$$
\begin{aligned}
E^{(3)} & =-\frac{i}{2}(\mathbf{r} \cdot \mathbf{v})\left(\mathbf{v} \cdot \nabla_{r}\right) \frac{(\mathbf{r} \cdot \mathbf{v})}{r} V^{(0)^{\prime}}(r) \\
& =\frac{i}{2}\left(\mathbf{v} \cdot \boldsymbol{\nabla}_{r}\right) \mathbf{v}^{j}\left\{\delta^{j k} V^{(0)}(r)-\frac{\mathbf{r}^{j} \mathbf{r}^{k}}{r} V^{(0)^{\prime}}(r)\right\} \mathbf{v}^{k} .
\end{aligned}
$$

We next consider the expressions which contain insertions with three fields, namely $E_{3}^{(1)}, E_{3}^{(2)}$ and the three terms in $E^{(4)}$. In $E_{11}^{(4)}$ we can symmetrize the factor in front with respect to $t^{\prime}$ and $t^{\prime \prime}$, i.e., replace $\left(t^{\prime}-t^{\prime \prime}\right)\left(t^{\prime \prime \prime}-t^{\prime \prime}\right) \rightarrow\left(t^{\prime}\right.$ $\left.-t^{\prime \prime}\right)^{2} / 2$. Then one has $E_{3}^{(1)}+E_{11}^{(4)}=0$. In $E_{3}^{(2)}$ and $E_{22}^{(4)}$ we exchange the coordinates $\mathbf{x}_{1}$ and $\mathbf{x}_{2}$ by applying $C P$, furthermore we exchange the integration variables $t^{\prime \prime}$ and $t^{\prime \prime \prime}$. The remaining three terms then all involve the same correlator. Symmetrizing with respect to $t^{\prime}, t^{\prime \prime}$ we get

$$
\begin{aligned}
E_{3}^{(2)}+E_{22}^{(4)}-2 E_{12}^{(4)} & =\lim _{T \rightarrow \infty} \frac{3 i}{4 T} \int_{-T / 2}^{T / 2} d t^{\prime} \int_{-T / 2}^{T / 2} d t^{\prime \prime} \int_{-T / 2}^{T / 2} d t^{\prime \prime \prime}\left(t^{\prime}-t^{\prime \prime}\right)^{2}\left\langle\left\langle g \mathbf{v} \cdot \mathbf{E}\left(\mathbf{x}_{1}, t^{\prime}\right) g \mathbf{v} \cdot \mathbf{E}\left(\mathbf{x}_{1}, t^{\prime \prime}\right) g \mathbf{v} \cdot \mathbf{E}\left(\mathbf{x}_{2}, t^{\prime \prime \prime}\right)\right\rangle\right\rangle_{c} \\
& =\lim _{T \rightarrow \infty} \frac{3}{4 T}\left(\mathbf{v} \cdot \nabla_{\mathbf{x}_{2}}\right) \int_{-T / 2}^{T / 2} d t^{\prime} \int_{-T / 2}^{T / 2} d t^{\prime \prime}\left(t^{\prime}-t^{\prime \prime}\right)^{2}\left\langle\left\langle g \mathbf{v} \cdot \mathbf{E}\left(\mathbf{x}_{1}, t^{\prime}\right) g \mathbf{v} \cdot \mathbf{E}\left(\mathbf{x}_{1}, t^{\prime \prime}\right)\right\rangle\right\rangle_{c} \\
& =3 i\left(\mathbf{v} \cdot \nabla_{r}\right) \mathbf{v}^{j} T^{j k} \mathbf{v}^{k} .
\end{aligned}
$$


In the third line we wrote the insertion $\int \cdots i g \mathbf{v}$ $\cdot \mathbf{E}\left(\mathbf{x}_{2}, t^{\prime \prime}\right) d t^{\prime \prime}$ as differentiation of the loop with respect to $\mathbf{x}_{2}$, in the last line we introduced the definition of $T^{j k}$ in Eq. (49). Lorentz invariance requires that the sum of Eqs. (67), (68), and (69) vanishes. This gives

$$
i\left(\mathbf{v} \cdot \nabla_{r}\right) \mathbf{v}^{j}\left\{S^{j k}+2 T^{j k}+\frac{\delta^{j k}}{2} V^{(0)}(r)-\frac{\mathbf{r}^{j} \mathbf{r}^{k}}{2 r} V^{(0)^{\prime}}(r)\right\} \mathbf{v}^{k}=0 .
$$

The term in curly brackets is just the expression which has to vanish according to Eqs. (23) and (24) and [13].

Equation (70) is only slightly weaker than the statement of the vanishing of the curly bracket. Let us introduce the combinations

$$
\begin{aligned}
& f(r)=V_{d}(r)+\frac{1}{2} V_{b}(r)+\frac{1}{4} V^{(0)}(r)-\frac{r}{12} V^{(0)^{\prime}}(r), \\
& g(r)=V_{e}(r)+\frac{1}{2} V_{c}(r)+\frac{r}{4} V^{(0)^{\prime}}(r) .
\end{aligned}
$$

These are the functions that have to vanish according to Eqs. (23) and (24) and [13] if $S^{j k}$ and $T^{j k}$ are decomposed into $V_{b}(r), \ldots, V_{e}(r)$. In our case Eq. (70) gives two differential equations which appear as factors of $4 i \mathrm{v}^{2}(\mathbf{r} \cdot \mathbf{v}) / r^{2}$ and $4 i(\mathbf{r} \cdot \mathbf{v})^{3} / r^{4}$, respectively. They read

$$
r f^{\prime}(r) / 2-g(r)+r g^{\prime}(r) / 6=0, \quad g(r)-r g^{\prime}(r) / 2=0 .
$$

The general solution is $g(r)=a r^{2}, f(r)=2 a r^{2} / 3+b$ with $a, b=$ const. Only $a=0$ appears physically reasonable, while the constant $b$ may be reabsorbed into the static potential, after suitable modification of the matching to pNRQCD.

\section{CONCLUSIONS}

In this work we have derived identities among the potentials of pNRQCD (as given in Sec. II) either by direct application of the Poincaré algebra to the potentials in their general form or by explicit Lorentz transformation of the potentials in terms of Wilson loops. In this way we have proved that the identities found long ago for the spindependent [12] and spin-independent potentials [13] are correct, despite several subtleties involved in the manipulation of Wilson loops overlooked by the literature (as first pointed out in [2] and discussed in Appendix B). We have thereby also proved that Lorentz invariance is the reason behind these two sets of identities, providing a unified framework.

From the general arguments of Sec. III the identities (21)-(25) are the only ones that can be derived, between the potentials up to order $1 / \mathrm{m}^{2}$. The identities that could constrain the potentials of order $1 / m$ and the momentum independent $1 / \mathrm{m}^{2}$ potentials would involve $1 / \mathrm{m}^{3}$ potentials, which are, at present, unknown. In this respect the "brute force" method used in Sec. IV may be more useful. It may be used in order to constrain Wilson loop operators that are relevant for the calculation of the potentials up to order $1 / \mathrm{m}^{2}$, but do not necessarily show up as potentials themselves. In particular, one could apply transformations similar to those discussed in Secs. IV A and IV B on Wilson loops with two or more field insertions. This would result in constraints involving Wilson loops with three or more field insertions. These types of Wilson loops are known to contribute to the momentum-independent potentials of order $1 / \mathrm{m}^{2}$ [2].

The complementarity of the two methods discussed in this work, which, however, are both applications of Poincare invariance, can be made clear by noticing that the identities (21) and (22) proved in Sec. III actually differ from the identity (47) proved in Sec. IV A. Indeed, combining Eq. (47) with Eqs. (21) and (22) we get $c_{S}^{(j)}=2 c_{F}^{(j)}-1$, which, therefore, may be derived in this way without explicitly using reparametrization invariance arguments on the fields of the heavy quark effective field theory Lagrangian.

Finally, we comment on the power-counting issue mentioned in the Introduction. Equations (21)-(24) contain combinations of $1 / \mathrm{m}^{2}$ potentials and the static potential. Since $V^{(0)}$ scales like $m v^{2}$ in the heavy quark velocity $v$, as usual in non-relativistic bound states, then the combinations of potentials of dimension 3 appearing in Eqs. (21) and (22) must scale as $m^{3} v^{4}$ and those of dimension 1 appearing in Eqs. (23) and (24) as $m v^{2}$. This result is not trivial. It implies that the considered potentials are suppressed by an extra power of $v$ with respect to the "natural" power counting based on their dimension. In perturbative QCD this extra suppression factor is typically provided by the coupling constant, $\alpha_{\mathrm{s}} \sim v$. Poincaré invariance tells that an extra suppression factor $v$ must be dynamically generated also in the non-perturbative regime.

\section{ACKNOWLEDGMENTS}

N.B. and A.V. thank the Institut für Theoretische Physik of the University of Heidelberg for the warm hospitality and the Alexander von Humboldt Foundation for support during the first stage of this work.

\section{APPENDIX A: POTENTIALS AND LORENTZ TRANSFORMATIONS}

In this section we show in more detail how the dependence on $\mathbf{U}^{(0)}, \mathbf{U}^{(1,0)}$ and $\mathbf{U}^{(0,1)}$ cancels in the final relations (21)-(25). In order to be definite we will assume

$$
\begin{aligned}
\mathbf{U}^{(0)}(\mathbf{r}) & =U^{(0)}(r) \mathbf{r}, \\
\mathbf{U}^{(1,0)}\left(\mathbf{r}, \mathbf{p}_{1}\right) & =\left\{g(r) \frac{\mathbf{r}}{r}, \mathbf{p}_{1}^{2}\right\}+\left\{f(r), \mathbf{p}_{1}\right\}+h(r) \mathbf{r}, \\
\mathbf{U}^{(0,1)}\left(\mathbf{r}, \mathbf{p}_{2}\right) & =\left\{g(r) \frac{\mathbf{r}}{r}, \mathbf{p}_{2}^{2}\right\}+\left\{f(r), \mathbf{p}_{2}\right\}+h(r) \mathbf{r},
\end{aligned}
$$

where $g, f$ and $h$ are arbitrary functions. From Eq. (14) it follows that one must have $g(r)=0$. Inserting Eqs. (19), (18), (17) in Eq. (12) we get, up to order $1 / m$, the following five equations: 


$$
\begin{gathered}
r \frac{d}{d r} v_{b}^{(0)}(r)+2 V_{\mathbf{L}^{2}}^{(2,0)}(r)-V_{\mathbf{L}^{2}}^{(1,1)}(r)-r \frac{d}{d r} U^{(0)}(r)=0 \\
r \frac{d}{d r} v_{a}^{(0)}(r)+2 V_{\mathbf{L}^{2}}^{(0,2)}(r)-V_{\mathbf{L}^{2}}^{(1,1)}(r)+r \frac{d}{d r} U^{(0)}(r)=0 \\
-v_{a}^{(0)}(r)-2 V_{\mathbf{L}^{2}}^{(2,0)}(r)+V_{\mathbf{L}^{2}}^{(1,1)}(r)-2 V_{\mathbf{p}^{2}}^{(2,0)}(r)+V_{\mathbf{p}^{2}}^{(1,1)}(r) \\
-U^{(0)}(r)=0, \\
-v_{b}^{(0)}(r)-2 V_{\mathbf{L}^{2}}^{(0,2)}(r)+V_{\mathbf{L}^{2}}^{(1,1)}(r)-2 V_{\mathbf{p}^{2}}^{(0,2)}(r)+V_{\mathbf{p}^{2}}^{(1,1)}(r) \\
+U^{(0)}(r)=0, \\
\frac{f(r)}{r} \frac{d}{d r} V^{(0)}(r)=0 .
\end{gathered}
$$

Adding Eq. (A4) to Eq. (A5) and Eq. (A6) to Eq. (A7) we get Eqs. (23) and (24). Subtracting them and combining the result, we get Eq. (25). If $d V^{(0)} / d r \neq 0$, Eq. (A8) gives

$$
f(r)=0,
$$

therefore, it follows that $\mathbf{U}^{(1,0)}$ and $\mathbf{U}^{(0,1)}$ do not depend linearly on $\mathbf{p}_{1}$ and $\mathbf{p}_{2}$ respectively. Elsewhere, if $d V^{(0)} / d r=0, f$ may stay arbitrary; this, however, does not spoil the validity of the relations (23)-(25). The above derivation may be extended to more general functions $\mathbf{U}^{(1,0)}$ and $\mathbf{U}^{(0,1)}$ that admit a power expansion in the momenta.

\section{APPENDIX B: SUBTLETIES AND INCORRECT PROOFS}

In this section we clarify a false statement, due to a wrong treatment of end-point string contributions, which can be found in the literature. Let us consider the function [10-12]

$$
\begin{aligned}
V_{2}^{\prime}(r)= & \frac{i}{2} \epsilon^{k i j} \frac{\mathbf{r}^{k}}{r} \lim _{T \rightarrow \infty} \frac{1}{T} \int_{-T / 2}^{T / 2} d t \int_{-T / 2}^{T / 2} d t^{\prime \prime}\left(t-t^{\prime \prime}\right) \\
& \times\left\langle\left\langle g \mathbf{B}^{i}\left(\mathbf{x}_{1}, t^{\prime \prime}\right) g \mathbf{E}^{j}\left(\mathbf{x}_{2}, t\right)\right\rangle\right\rangle,
\end{aligned}
$$

related to the spin-orbit potentials introduced in Sec. IV through the relation $c_{F}^{(1)} V_{2}^{\prime}=r V_{L_{2} S_{1}}^{(1,1)}$. In the review [6] one finds the statement that the factor $t^{\prime \prime}$ in the integrand may be dropped, i.e., that the value of $V_{2}^{\prime}$ does not change if we replace $\left(t-t^{\prime \prime}\right)$ with $t$. Indeed, in this second form the potential $V_{2}^{\prime}$ can be found, for instance, in $[10,11]$. It has been pointed out and proved in [2] that this statement is wrong

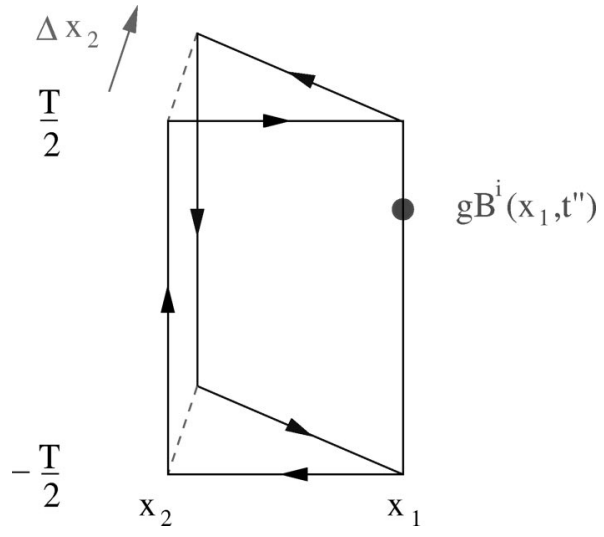

FIG. 4. Difference between the expression (40) shifted accordingly to $\mathbf{x}_{2} \rightarrow \mathbf{x}_{2}+\Delta \mathbf{x}_{2}$ and the original one. The arrows indicate the orientation of the loops, which arise after expanding up to first order of $\Delta \mathbf{x}_{2}$. The corners of the old and the new loop have been connected by dashed lines, in order to visualize the form of the infinitesimal loops, which correspond to the insertions of chromoelectric (time-like rectangle) and chromomagnetic fields (space-like triangles at $\pm T / 2$ )

even at leading order in perturbation theory. In the following we will make clear how it was possible to arrive at that erroneous statement.

Let us start from the identity (40). Applying the differential operator $\boldsymbol{\nabla}_{\mathbf{x}_{2}}^{j}$ to it and writing the differentiation of the time-like lines as insertions of chromoelectric fields and the differentiation of the space-like lines as insertions of chromomagnetic fields, we obtain

$$
\begin{aligned}
& i \int_{-T / 2}^{T / 2} d t\left\langle\left\langle g \mathbf{B}^{i}\left(\mathbf{x}_{1}, t^{\prime \prime}\right) g \mathbf{E}^{j}\left(\mathbf{x}_{2}, t\right)\right\rangle\right\rangle-i \boldsymbol{\epsilon}^{j k l} \mathbf{r}^{k} \int_{0}^{1} d s(1-s) \\
& \quad \times\left\langle\left\langle g \mathbf{B}^{l}\left(\mathbf{x}_{2}+s \mathbf{r}, T / 2\right) g \mathbf{B}^{i}\left(\mathbf{x}_{1}, t^{\prime \prime}\right)\right\rangle\right\rangle+i \boldsymbol{\epsilon}^{j k l} \mathbf{r}^{k} \\
& \quad \times \int_{0}^{1} d s(1-s)\left\langle\left\langle g \mathbf{B}^{i}\left(\mathbf{x}_{1}, t^{\prime \prime}\right) g \mathbf{B}^{l}\left(\mathbf{x}_{2}+s \mathbf{r},-T / 2\right)\right\rangle\right\rangle=0 .
\end{aligned}
$$

Assuming that the last two lines, once inserted in Eq. (B1), vanish in the large $T$ limit (as tacitly assumed by previous authors), leads immediately to the conclusion that the factor $t^{\prime \prime}$ may, indeed, be dropped in the integrand of Eq. (B1).

The key point is that, as argued in [2], the end-point string contributions, corresponding to the small triangles at time $\pm T / 2$ of Fig. 4, do not vanish in the large $T$ limit in Eq. (B1). This is easy to check at lowest order of perturbation theory, where we have

$$
\begin{array}{r}
i \int_{-T / 2}^{T / 2} d t\left\langle\left\langle g \mathbf{B}^{i}\left(\mathbf{x}_{1}, t^{\prime \prime}\right) g \mathbf{E}^{j}\left(\mathbf{x}_{2}, t\right)\right\rangle\right\rangle=-2 i \frac{C_{f} \alpha_{\mathrm{s}}}{\pi} \epsilon^{i j l} \mathbf{r}^{l}\left(\frac{1}{\left[\left(t^{\prime \prime}-T / 2\right)^{2}-r^{2}-i \epsilon\right]^{2}}-\frac{1}{\left[\left(t^{\prime \prime}+T / 2\right)^{2}-r^{2}-i \epsilon\right]^{2}}\right), \\
i \epsilon^{j k l} \mathbf{r}^{k} \int_{0}^{1} d s(1-s)\left\langle\left\langle g \mathbf{B}^{i}\left(\mathbf{x}_{1}, t^{\prime \prime}\right) g \mathbf{B}^{l}\left(\mathbf{x}_{2}+s \mathbf{r},-T / 2\right)\right\rangle\right\rangle \underset{\text { pert }}{=}-2 i \frac{C_{f} \alpha_{\mathrm{s}}}{\pi} \epsilon^{i j l} \mathbf{r}^{l} \frac{1}{\left[\left(t^{\prime \prime}+T / 2\right)^{2}-r^{2}-i \epsilon\right]^{2}},
\end{array}
$$




$$
i \epsilon^{j k l} \mathbf{r}^{k} \int_{0}^{1} d s(1-s)\left\langle\left\langle g \mathbf{B}^{l}\left(\mathbf{x}_{2}+s \mathbf{r}, T / 2\right) g \mathbf{B}^{i}\left(\mathbf{x}_{1}, t^{\prime \prime}\right)\right\rangle\right\rangle=-2 i \frac{C_{f} \alpha_{\mathrm{s}}}{\pi} \epsilon^{i j l} \mathbf{r}_{\text {pert }}^{l} \frac{1}{\left[\left(t^{\prime \prime}-T / 2\right)^{2}-r^{2}-i \epsilon\right]^{2}},
$$

with $C_{f}=\left(N_{c}^{2}-1\right) / 2 N_{c}=4 / 3$ in QCD. It is clear that all three lines of Eq. (B2) give the same kind of contribution in leading order perturbation theory and that the identity is satisfied only if all three pieces are taken into account.

The argument can be extended to any order of perturbation theory. If we analyze the above end-point string contributions by decomposing them into the eigenstates $|n\rangle^{(0)}$ of the static NRQCD Hamiltonian, we obtain for $T \rightarrow \infty$

$$
\begin{gathered}
\frac{i}{T} \int_{-T / 2}^{T / 2} d t^{\prime \prime} t^{\prime \prime}\left[\left\langle\left\langle g \mathbf{B}^{i}\left(\mathbf{x}_{1}, t^{\prime \prime}\right) g \mathbf{B}^{l}\left(\mathbf{x}_{2}+s \mathbf{r},-T / 2\right)\right\rangle\right\rangle-\left\langle\left\langle g \mathbf{B}^{l}\left(\mathbf{x}_{2}+s \mathbf{r}, T / 2\right) g \mathbf{B}^{i}\left(\mathbf{x}_{1}, t^{\prime \prime}\right)\right\rangle\right\rangle\right] \\
=\frac{1}{a_{0}^{2}} \sum_{n \neq 0} \frac{a_{n} \mathbf{b}_{0}^{l(0)}\left\langle n\left|g \mathbf{B}^{i}\left(\mathbf{x}_{1}\right)\right| 0\right\rangle^{(0)}-a_{0} \mathbf{b}_{n}^{l(0)}\left\langle 0\left|g \mathbf{B}^{i}\left(\mathbf{x}_{1}\right)\right| n\right\rangle^{(0)}}{E_{n}^{(0)}-E_{0}^{(0)}}+\mathcal{O}\left(\frac{1}{T}\right)
\end{gathered}
$$

with the same notation as in Sec. IV A. The ground state $|0\rangle^{(0)}$ has no overlap with the state made from two static quarks and a chromomagnetic field $g \mathbf{B}^{i}\left(\mathbf{x}_{2}+s \mathbf{r},-T / 2\right)$ connected by a straight string, hence $\mathbf{b}_{0}^{l}=0$. However, contrary to Eq. (46), there are still contributions that do not vanish for $T \rightarrow \infty$. This general argument definitely proves that the end-point string contributions of Eq. (B2) do not vanish and, therefore, we cannot drop the factor $t^{\prime \prime}$ in Eq. (B1).

So, we finally find that a forgotten contribution corrects a wrong proof where necessary, but cancels in a well-known relation [Eq. (47)], where it would be very disturbing. Future manipulations of the above type should, therefore, be done with the appropriate care, and old ones revisited appropriately.

[1] N. Brambilla, A. Pineda, J. Soto, and A. Vairo, Phys. Rev. D 63, 014023 (2001).

[2] A. Pineda and A. Vairo, Phys. Rev. D 63, 054007 (2001).

[3] K. G. Wilson, Phys. Rev. D 10, 2445 (1974).

[4] G. S. Bali, Phys. Rep. 343, 1 (2001); G. S. Bali, A. Wachter, and K. Schilling, Phys. Rev. D 56, 2566 (1997).

[5] N. Brambilla and A. Vairo, Phys. Rev. D 55, 3974 (1997); M. Baker, J. S. Ball, N. Brambilla, and A. Vairo, Phys. Lett. B 389, 577 (1996); M. Baker, J. S. Ball, N. Brambilla, G. M. Prosperi, and F. Zachariasen, Phys. Rev. D 54, 2829 (1996); N. Brambilla, P. Consoli, and G. M. Prosperi, ibid. 50, 5878 (1994); for a general introduction, see, e.g., N. Brambilla and A. Vairo, in Strong Interactions at Low and Intermediate Energies, edited by J. L. Goity (World Scientific, Singapore, 2000), p. 151, hep-ph/9904330.

[6] W. Lucha, F. Schöberl, and D. Gromes, Phys. Rep. 200, 127 (1991).

[7] G. Bali [4].

[8] W. E. Caswell and G. P. Lepage, Phys. Lett. 167B, 437 (1986); G. T. Bodwin, E. Braaten, and G. P. Lepage, Phys. Rev. D 51, 1125 (1995); 55, 5853(E) (1997).

[9] Y. Chen, Y. Kuang, and R. J. Oakes, Phys. Rev. D 52, 264
(1995); N. Brambilla and A. Vairo, Nucl. Phys. B (Proc. Suppl.) 74, 210 (1999).

[10] E. Eichten and F. Feinberg, Phys. Rev. D 23, 2724 (1981).

[11] A. Barchielli, E. Montaldi, and G. M. Prosperi, Nucl. Phys. B296, 625 (1988); B303, 752(E) (1988).

[12] D. Gromes, Z. Phys. C 26, 401 (1984).

[13] A. Barchielli, N. Brambilla, and G. Prosperi, Nuovo Cimento A 103, 59 (1990).

[14] Y. Chen and Y. Kuang, Z. Phys. C 67, 627 (1995).

[15] S. Fleming, I. Z. Rothstein, and A. Leibovich, Phys. Rev. D 64, 036002 (2001)

[16] A. Pineda and J. Soto, Nucl. Phys. B (Proc. Suppl.) 64, 428 (1998); N. Brambilla, A. Pineda, J. Soto, and A. Vairo, Nucl. Phys. B566, 275 (2000).

[17] L. L. Foldy, Phys. Rev. 122, 275 (1961).

[18] R. A. Krajcik and L. L. Foldy, Phys. Rev. D 10, 1777 (1974).

[19] K. J. Sebastian and D. Yun, Phys. Rev. D 19, 2509 (1979).

[20] P. A. M. Dirac, Rev. Mod. Phys. 21, 392 (1949).

[21] A. V. Manohar, Phys. Rev. D 56, 230 (1997).

[22] Yu. A. Simonov, Yad. Fiz. 50, 213 (1989) [Sov. J. Nucl. Phys. 50, 134 (1989)]. 